\title{
The features of antigen prevalence of Rhesus system in donor population
}

\author{
M. Nagervadze ${ }^{1}$, L. Akvlediani ${ }^{2}$, I. Tsintsadze ${ }^{2}$, T. Koiava ${ }^{1}$, R. Loria ${ }^{3}$, S. Tskvitinidze ${ }^{1}$, \\ R. Khukhunaishvili ${ }^{1}$, M. Koridze ${ }^{1}$ \\ ${ }^{1}$ Department of Biology, Batumi Shota Rustaveli State University, Batumi, Georgia \\ ${ }^{2}$ BAU International University of Batumi, Batumi, Georgia \\ ${ }^{3}$ Diagnostic Laboratory of "Health Centre Medina Ltd" of \\ Batumi, Georgia
}

Received: October 13, 2020. Revised: March 29, 2021. Accepted: April 12, 2021. Published: April 15, 2021.

\begin{abstract}
Research materials and methods. 852 voluntary Georgian blood donors have been typed on red blood cells group antigens. The research materials have taken from the diagnostic laboratory of Health Centre of Batumi (Georgia republic). The immunoserological methods with monoclonal anti -AB, -B, -A, A1, -A2 (H), -C, -c, - D, -E, e (Bio-Rad, cypress diagnostics) antibodies was used for typing blood. The ID cards, such as $\mathrm{ABO} / \mathrm{D}{ }^{+}$Reverse Grouping (Bio-Rad) were also used for typing of erythrocyte antigens.
\end{abstract}

Result. Prevalence of $\mathrm{Rh}$ system antigens in the studied group is looks like so: e antigens - $94,6 \%$, c antigens $85 \%$, C-68,03, E antigens - 38,07\%. The majority (84\%) of the studied donors are Rh-positive $(n=719), 133(16 \%)$ donors are Rh-negative. $C$ antigen most common is present in the combination with $D$ antigen. $65,8 \%$ case donors had CD+ combination $(n=561)$. $E$ antigen in most cases is presented with a combination of $D$ antigen. $36,9 \%$ of the studied donors $(n=306)$ had ED+ combination. A miserable number of studied donors had CD - $(2,23 \%$; $n=19)$ and ED - $(1,17 \% ; n=9)$ combinations. We have studied the $\mathrm{Rh}$ phenotypes prevalence in blood donors. According to RHD, RHC, and

RHE gene loci, there are 18 theoretically possible phenotypical groups. Among them half (nine) are Rhpositive and the rest of them are Rh-negative. The Rhpositive phenotypes are: $\mathrm{CDE}$; CDEe; CDe; CcDE; CcDEe; CcDe; ccDE; cDEe and cDe. Rh-negative phenotypes are CdE; CdEe; Cde; CcdE; CcdEe; Ccde; cdE; cdEe; cde. We allocated $17 \mathrm{Rh}$ phenotypes among studied donors. Only one phenotype $\mathrm{CdE}$, which belongs to Rh negative group, was not present in studied donors. Other 17 phenotypes showed different frequencies. Some of them were only in a single case, for example, cdEe, cdE, CdEe phenotypes had only one donor. The majority of the phenotype in he studied donors $(27,8 \pm 1,53 \%)$ was CcDe $(n=237) . \quad$ CcDEe - 19,3 $\pm 1,35 \%(n=165) ; 125$ donors have CDe phenotype $(14,6 \pm 1,2)$; The frequency of cde was $13,1 \pm 1,5 \%$, which means that 112 studied donors belonged to this phenotype group; 87 studied donors had cDEe phenotype characteristics $(10,2 \%)$; The frequency of $\mathrm{cDe}$ was 4,9\% $(n=42) ; 19$ donors had CDEe phenotype. Other phenotypes (CDE, Cde, CcdEe, Ccde) frequency was very low.

Conclusion. Our studied donors are characterized by rather high polymorphism. The Georgian donor's population is heterogenic, especially high heterogeneity are shown in $R h$ positive phenotypes. The obtained data is vital importance for the preparation of whole blood or certain blood components for the purpose of their rational usage in blood transfusion.

Kay words: Rh system, Phenotype, blood donor, erythrocytes, antigen.

\section{INTRODUCTION}

Group antigens determine the trophic and regulatory functions of blood cells. It is well known that they are part of cellular receptors, through which hormones, vitamins, enzymes, and other proteins are transported in the circulatory system and are the main structural elements of cell membrane adhesion [1] [2] [3]. Blood group antigens determine the adaptation of a human to the environment. Erythrocyte group systems are significant for ethnic anthropology. The combination of antigens of group systems in the human population is likely a result of balanced polymorphism. According to the erythrocyte group antigens, the correlation between balanced polymorphism and various types of infectious and noninfectious diseases was well established [4] [5] [6] [7]. 
blood group antigens have ability to initiate creation of antierythrocyte antibodies, which can damage the red blood cells. The antierythrocyte antibodies cause different types of transfusion reactions, post-transfusion difficulties and hemolytic disease in newborns [8] [9].

Currently, about 39 systems of erythrocyte groups are distinguished, which include about 346 antigens that can induce the severe transfusion reactions. From a clinical point of view, ABO, Rh, Kell, MNSs, and other systems are the most and vitally important.

Blood erythrocyte group antigens are the genetically strictly deterministic feature. In spite of resistant specific characteristics, the relevant hereditary factors within the ranges of species at the level of populations and individuals are characterized with rather high polymorphism [10] [11] [12] [13].

The Rhesus system consists of 49 defined antigens. Five Rh system antigens (D, C, c, E, and e) are the cause of the second most important adverse effects, after those due to ABO, when group incompatibility occurs in blood transfusion. Their function in evolution, genetic disease, and forensic medicine is being intensively investigated. The rhesus system D, C, c, E, and e antigens are determined by two genes RHD and RHCE encoding for $\mathrm{RhD}$ and RhCE proteins, respectively [14] [15].

The Rhesus blood group systems show considerable genetic variation between populations across the world. Many blood transfusions and blood banks from different countries and regions have many investigations about study the prevalence of Rh antigens [10] [12] [16] [17] [18].

Our goal was to study the $\mathrm{Rh}$ antigens $(\mathrm{C}, \mathrm{c}, \mathrm{D}, \mathrm{E}, \mathrm{e})$ in the Georgian donor population. The only rhesus system D antigen has typing in donor-recipient throughout the region (Adjara, Georgia republic). It is interesting to find the frequency of other $\mathrm{Rh}$ antigens and $\mathrm{Rh}$ phenotypes and estimate the theoretical risk of sensibilization caused by Rh antigen.

Our research also has clinical applications. Based on our research clinic has the possibility to have its donors' $\mathrm{Rh}$ phenotypes and combinations database. By using this database, it is quite easy to select fully compatible donors for recipients. This data is vitally important for patients with rare Rh phenotype combinations. A similar approach would certainly decrease the risks of post transfusion reactions caused by rare antigens.

The erythrocyte $\mathrm{Rh}$ blood group system shows high polymorphism and heterogeneity with different populations. Our study shows Rh blood group phenotype polymorphism in Georgian blood donor population.

\section{MATERIAL AND METHODS}

The subjects of our study are blood donors. The material of the research is vein blood samples. 852 donors have been typing on red blood cells' antigens. The majority of our studied donors were males (627 are male and 225 female). The most of our studied donors (92,5\%) are Georgians, but there are some people from Armenia, Azerbaijan, Ossetia, Russia,
Ukraine, India and others. The research was carried out 20162019 years.

According to the recommended norms and regulations of World Health Organization (WHO), only people from a certain age group can donate blood. The age range of the observed donors is 18-60 (The average age of the studied blood donors is 38 years). The minimum weight of the donors is $50 \mathrm{~kg}$ (The average weight of the studied blood donors is 65 $\mathrm{kg}$ ). One of the most necessary factors for blood donation is also the level of hemoglobin in the blood. Hemoglobin level for the male donors should be at least $130.0 \mathrm{mg}$ all and for the female donors $120 \mathrm{~g}$.

The material for the research has been taken adhering to the bioethics norms. Based on the conclusion of the Clinic Bioethics Committee, we were able to use the blood materials from the clinic's laboratory. We did not take an additional invasion for collecting blood samples from the donors and don't contact with donors. The studying materials were provided to Batumi health Center Medina Ltd. Laboratory analysis was carried out on the basis of the laboratory of immunogenetics at Batumi Shota Rustaveli State University (BSU).

The research methods were immuneserologically forward and reversed methods with monoclonal antibodies. We used following monoclonal antibodies: anti -AB, -B, -A, A1, -A2 (H), -C, -c, - D, -E, -e (Bio-Rad, cypress diagnostics). We used as a plate as tube methods during research.

In addition, the studied donor group in some cases was also determined by the column agglutination methods. Gel card system-so-called ID cards were used (Bio-Rad). In particular, special ID cards $\mathrm{ABO} / \mathrm{Rh}$ (A, B, DVI+/A, B, DVI+) for donors and A1, A2, B/I, II, III for reverse phenotyping of the ABO system were used during research. Some Rh- blood donors additionally were typing for weak D (ID-Diaclon AntiD for weak D, Bio-Rad).

All the research methods were based on specific antigenantibody agglutination reaction. All negative reactions (without agglutination) were confirmed microscopically. We used an optic microscope with different magnification (10X4, $10 \mathrm{X} 10$, and 10X100). This method helps us to identify lowlevel agglutination reactions.

The $\mathrm{Rh}$ antigens, phenotypes and antigens combinations frequencies were expressed as percentages. This percentage is calculated by summing the number of donors, which contains the specific erythrocyte antigen, phenotype or antigens combination and dividing by the total number of studying materials. For calculated the $\mathrm{Rh}$ system allele frequency we used The Hardy-Weinberg equation. The frequency of Rh haplotype was calculated by using the formula shown in the table VI. We used the on-variable chi-square general formula for calculating chi-square $\left(\chi^{2}\right)$. The level of statistical significance was set at 0.05 . 


\section{RESULT}

852 voluntary Georgian blood donors have been typed on red blood cells group antigens. As already was mentioned above The $\mathrm{Rh}$ group system consists of 49 defined blood group antigens [19] [20]. The five antigens D, C, c, E, and e are the vital important for transfusion. The study of Rh blood group antigens, phenotype, and $\mathrm{Rh}$ antibodies are very useful in routine and advanced clinical practice in blood transfusion centers. We study the prevalence of these five Rh antigens in Georgian blood donors $(\mathrm{n}=852)$ of both sex and different age (18-60 y.) (Table I).
The prevalence of Rh system antigens in the studied donors is looking like so: e antigen - 94,6\%; c antigen - $85 \%$; C$68,03 \%$; and $\mathrm{E}$ antigen - 38,07\%. The majority ( $84 \%$ ) of our studied donors are Rh-positive ( $\mathrm{n}=719)$, only $133(16 \%)$ donors are Rh-negative. We used one-variable chi-square criterion. The value of $\chi 2$ in the case is equal to 211,46 . These numbers are much higher than the critical value $(\mathrm{CV})$ of the criterion of the degree of freedom (d.f. $=3$ ), which is equal to 7,815 (Table I).

Table I . Prevalence and Chi-square analysis of Rhesus system antigens in the donors

\begin{tabular}{|c|c|c|c|c|c|}
\hline $\begin{array}{c}\text { Rh antigens } \\
\text { expressed } \\
\text { on cell }\end{array}$ & $\begin{array}{l}\text { Prevalence of } \\
\text { antigens }\end{array}$ & Df & $\chi^{2}$ & $\mathbf{C V}$ & $P$ \\
\hline $\mathrm{C}$ & $68,03 \% \pm 1,5$ & \multirow[t]{4}{*}{3} & \multirow[t]{4}{*}{211,46} & \multirow[t]{4}{*}{7,815} & \multirow{4}{*}{$\begin{array}{l}\text { The } \mathrm{P}-\text { Value is } \\
<.00001 . \text { The } \\
\text { result is } \\
\text { significant at } \mathrm{p} \\
<.05 \text {. }\end{array}$} \\
\hline $\mathrm{c}$ & $85 \% \pm 1,22$ & & & & \\
\hline $\mathrm{E}$ & $38,07 \% \pm 1,6$ & & & & \\
\hline e & $94,6 \% \pm 0.77$ & & & & \\
\hline
\end{tabular}

The frequency of distribution of $\mathrm{Rh}$ alleles in the studied donors was analyzed. Two alleles of the $\mathrm{RhC}$ gene occur with the following frequency: $\mathrm{C}-0,48, \mathrm{c}-0,54$. Allele's distribution in the studying population is equal to $1(\mathrm{p}+\mathrm{q}=1)$. For calculated the allele frequency we used The Hardy-
Weinberg equation. The distribution of two alleles of the RhE gene is as follows: $\mathrm{E}-0,61$, e $-0,40(\mathrm{p}+\mathrm{q}=1)$. RhD reveals a rather high frequency of distribution and it is 0,64 (Table II).

Table II. Frequency of distribution of alleles of the Rh system in donors.

\begin{tabular}{|c|c|c|}
\hline Rh system genes & calculated formula & Frequency \\
\hline $\mathrm{D}$ & $\boldsymbol{D}=1-\sqrt{d d}$ & 0.64 \\
\hline $\mathrm{C}$ & $\boldsymbol{C}=1-\sqrt{c c}$ & 0.48 \\
\hline $\mathrm{E}$ & $\boldsymbol{E}=1-\sqrt{e e}$ & 0.61 \\
\hline $\mathrm{c}$ & $\boldsymbol{c}=1-\sqrt{C C}$ & 0.54 \\
\hline $\mathrm{e}$ & $\boldsymbol{e}=1-\sqrt{E E}$ & 0.40 \\
\hline
\end{tabular}

As we see from Figure № $1 \mathrm{C}$ antigen most common is present in the combination with $\mathrm{D}$ antigen. $65,8 \%$ case we had $\mathrm{CD}(\mathrm{CRH}+)$ combination $(\mathrm{n}=561)$. A similar situation is with $\mathrm{E}$ antigen combination with $\mathrm{D}$ antigen. Erythrocyte $\mathrm{E}$ antigen in most donor cases is presented with a combination of $\mathrm{D}$ antigen (ED or $\mathrm{ERH}+$ ). 36, 9\% of the studied donors

$(n=306)$ had ED + combination. A miserable number of studied donors had CD - (CRH-) $(2,23 \% ; n=19)$ and $\mathrm{ED}-$ (ERH-) $(1,17 \% ; n=9)$ combinations. We can make the 
conclusion that $\mathrm{C}$ and $\mathrm{E}$ antigens are more linked with $\mathrm{D}$ antigens based on our data.

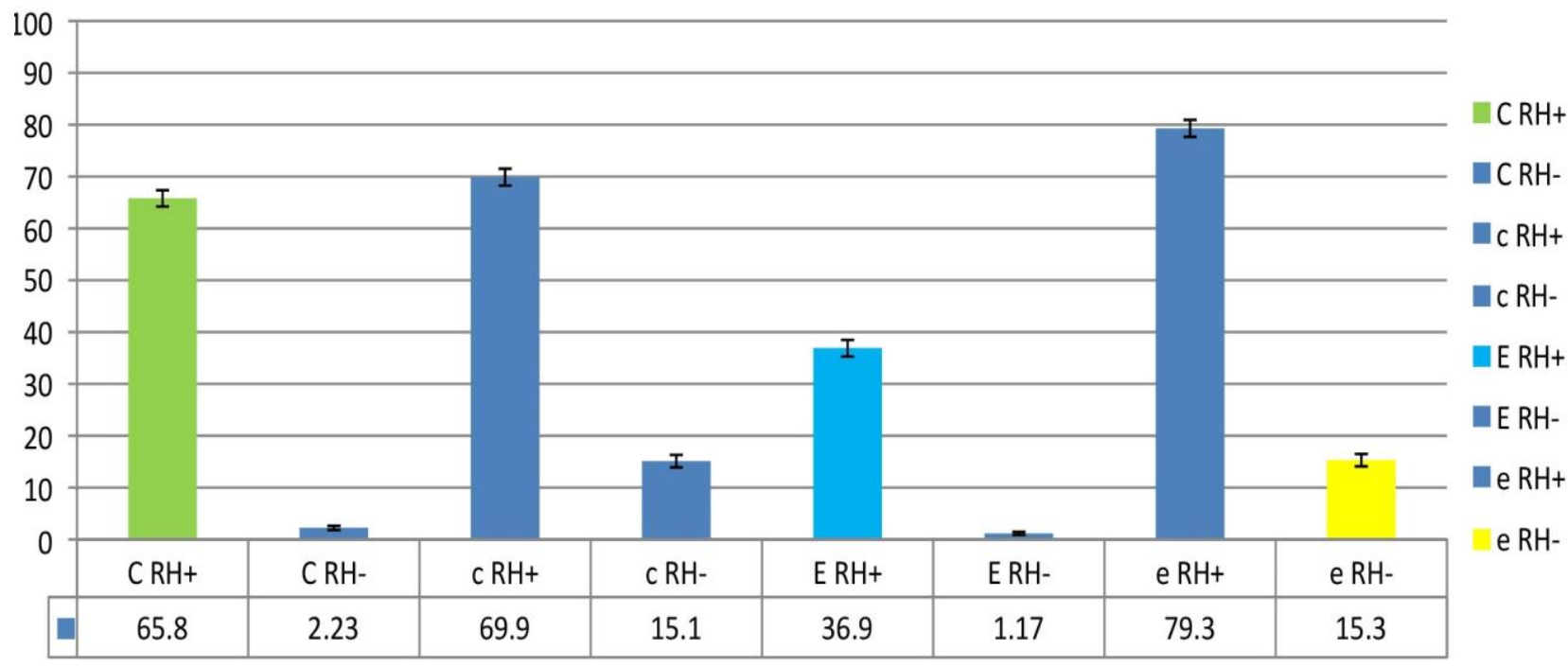

Figure № 1. $\mathrm{C}, \mathrm{c}, \mathrm{E}$ e antigens combination with $\mathrm{D}$ positive ( $\mathrm{Rh}+)$ and $\mathrm{D}$ negative ( $\mathrm{Rh}-)$ donors.

The Rh blood group system has two sets of nomenclatures . In our study, we used Fisher and Race CDE nomenclature [21]. We have studied the $\mathrm{Rh}$ phenotypes prevalence in blood donors. Phenotypic groups are divided according to serologically detected Rhesus system C, c, E, e, D antigens. For example phenotype $\mathrm{D}+\mathrm{C}+\mathrm{E}+\mathrm{c}-\mathrm{e}-$ means that donors blood have a positives reaction with anti-D, anti-C, and anti-E monoclonal antibodies, while anti-c and anti-e antibodies don't show any agglutination with donors' blood. $\mathrm{D}+\mathrm{C}+\mathrm{E}+\mathrm{c}-\mathrm{e}-$ phenotype also be written as $\mathrm{CDE}$. $\mathrm{D}+\mathrm{C}+\mathrm{E}+\mathrm{c}-\mathrm{e}+$ phenotype are written as $\mathrm{CDEe}$ and act.

According to RHD, RHC, and RHE gene loci there are 18 theoretically possible phenotypically groups. Among them, half (nine phenotypes) are Rh-positive and the rest of them are Rh-negative. The Rh-positive phenotypes are: CDE; CDEe; $\mathrm{CDe}$; $\mathrm{CcDE}$; $\mathrm{CcDE}$; $\mathrm{CcDe}$; ccDE; cDEe and cDe. Rh- negative phenotypes are CdE; CdEe; Cde; CcdE; CcdEe; Ccde; cdE; cdEe; cde. We allocated $17 \mathrm{Rh}$ phenotypes among studied donors. Only one phenotype $\mathrm{CdE}$, which belongs $\mathrm{Rh}$ negative group was not present in studied donors. Other 17 phenotypes showed different frequency (Table 3, Figure 2). Some of them were only in a single case, for example, cdEe, cdE, CdEe phenotypes had only one donor. Majority of the phenotype in the studied donors $(27,8 \pm 1,53 \%)$ was $\mathrm{CcDe}$ $(\mathrm{n}=237) . \quad \mathrm{CcDEe}-19,3 \pm 1,35 \%(\mathrm{n}=165) ; 125$ donors have CDe phenotype $(14,6 \pm 1,2)$; The frequency of cde was $13,1 \pm 1,5 \%$, which means that 112 studied donors belonged to this phenotype group; 87 studied donors had cDEe phenotype characteristics (10,2\%); The frequency of cDe was 4,9\% $(n=42) ; 19$ donors had CDEe phenotype. Other phenotypes (CDE, Cde, CcdEe, Ccde) frequency was very low (Table III, Figure 2).

Table III. The numbers of Rh phenotypes in the studied donors $(\mathrm{n}=852)$.

\begin{tabular}{|c|c|c|c|c|c|c|c|c|c|}
\hline Rh phenotype & $\begin{array}{l}\mathrm{O}(\mathrm{I}) \\
\mathrm{Rh}+\end{array}$ & $\begin{array}{l}\mathrm{O}(\mathrm{I}) \\
\mathrm{Rh}-\end{array}$ & $\begin{array}{l}\mathrm{A}(\mathrm{II}) \\
\mathrm{Rh}+\end{array}$ & $\begin{array}{l}\text { A(II) } \\
\text { Rh- }\end{array}$ & $\begin{array}{c}\mathrm{B}(\mathrm{III}) \\
\mathrm{Rh}+\end{array}$ & $\begin{array}{c}\text { B(III) } \\
\text { Rh- }\end{array}$ & $\begin{array}{c}\mathrm{AB}(\mathrm{IV}) \\
\mathrm{Rh}+\end{array}$ & $\begin{array}{c}\text { AB(IV) } \\
\text { Rh- }\end{array}$ & Total \\
\hline $\begin{array}{c}\text { CDE } \\
\mathbf{D}^{+} \mathbf{C}^{+} \mathbf{E}^{+} \mathbf{c}^{-} \mathbf{e}^{-}\end{array}$ & 3 & 0 & 2 & 0 & 0 & 0 & 0 & 0 & 5 \\
\hline $\begin{array}{c}\text { CDEe } \\
\mathbf{D}^{+} \mathbf{C}^{+} \mathbf{E}^{+} \mathbf{c}^{-} \mathbf{e}^{+}\end{array}$ & 6 & 0 & 9 & 0 & 3 & 0 & 1 & 0 & 19 \\
\hline $\begin{array}{c}\text { CDe } \\
\text { D }^{+} \mathbf{C}^{+} \mathbf{E}^{-} \mathbf{c}^{-} \mathrm{e}^{+}\end{array}$ & 64 & 0 & 48 & 0 & 12 & 0 & 1 & 0 & 125 \\
\hline $\begin{array}{c}\text { CcDEe } \\
\mathbf{D}^{+} \mathbf{C}^{+} \mathbf{E}^{+} \mathbf{c}^{+} \mathbf{e}^{+}\end{array}$ & 9 & 0 & 9 & 0 & 1 & 0 & 0 & 0 & 19 \\
\hline $\begin{array}{c}\text { CcD-ee } \\
\mathrm{D}^{+} \mathbf{C}^{+} \mathbf{E}^{+} \mathbf{c}^{-} \mathrm{e}^{+}\end{array}$ & 65 & 0 & 76 & 0 & 17 & 0 & 7 & 0 & 165 \\
\hline
\end{tabular}




\begin{tabular}{|c|c|c|c|c|c|c|c|c|c|}
\hline cDE & 125 & 0 & 84 & 0 & 20 & 0 & 8 & 0 & 237 \\
\hline cDEe & 11 & 0 & 7 & 0 & 1 & 0 & 1 & 0 & 20 \\
\hline ccD-ee & 52 & 0 & 22 & 0 & 9 & 0 & 4 & 0 & 87 \\
\hline CdE & 16 & 0 & 22 & 0 & 3 & 0 & 1 & 0 & 42 \\
\hline CCddEe & 0 & 0 & 0 & 0 & 0 & 0 & 0 & 0 & 0 \\
\hline 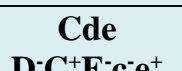 & 0 & 1 & 0 & 2 & 0 & 0 & 0 & 0 & 3 \\
\hline $\begin{array}{c}\text { CcddEE } \\
\mathbf{D}^{-} \mathbf{C}^{+} \mathbf{E}^{+} \mathbf{c}^{+} \mathbf{e}^{-}\end{array}$ & 0 & 1 & 0 & 0 & 0 & 0 & 0 & 0 & 1 \\
\hline $\begin{array}{c}\text { CcdEe } \\
\mathbf{D}^{-} \mathbf{C}^{+} \mathbf{E}^{+} \mathbf{c}^{+} \mathbf{e}^{+}\end{array}$ & 0 & 4 & 0 & 2 & 0 & 0 & 0 & 0 & 6 \\
\hline Ccde & 0 & 4 & 0 & 3 & 0 & 1 & 0 & 0 & 8 \\
\hline $\begin{array}{c}\mathbf{c d E} \\
\mathbf{D}^{-} \mathbf{C}^{-} \mathbf{E}^{+} \mathbf{c}^{+} \mathbf{e}^{-}\end{array}$ & 0 & 1 & 0 & 0 & 0 & 0 & 0 & 0 & 1 \\
\hline $\begin{array}{c}\text { cdEe } \\
D^{-} \mathbf{C}^{-} \mathbf{E}^{+} \mathbf{c}^{+} \mathrm{e}^{+}\end{array}$ & 0 & 0 & 0 & 1 & 0 & 0 & 0 & 0 & 1 \\
\hline Cde & 0 & 62 & 0 & 34 & 0 & 14 & 0 & 2 & 112 \\
\hline Total & 351 & 73 & 279 & 43 & 66 & 15 & 23 & 2 & 852 \\
\hline
\end{tabular}

There are some errors in determining the rhesus phenotype. Errors in determining the $\mathrm{Rh}$ factor is associated with a weak variation of the antigen $D$. According to the recommendations, additional studies should be carried out for all those cases where the Cde and cdE phenotypes are detected during the primary phenotyping of erythrocytes, since the weak D antigen is most often found together with the $\mathrm{C}$ or $\mathrm{E}$ antigen. The studied donors had three cases of the Cde phenotype and one case of the cdE phenotype. No weak D antigen was observed in any of these 4 cases and accordingly, the primary detected phenotype of rhesus did not changed.
$\mathrm{Rh}$ positive donors, as we already mentioned above have nine phenotypes and their frequency was quite different. The studied population is heterogenic based this charachteristics. Among Rh-positive donors two (CcDEe-22,9\% and $\mathrm{CcDe}-$ $32,9)$ phenotypes were spread with high frequency. The Majority $(55,8 \%)$ Rh-positive donors had these 2 phenotype characteristics on the erythrocyte. Another two phenotypes (CCDe - 17,38\% and cDEe -12,1\%) frequency equals $29,48 \%$. The Rest of the five phenotypes (CDE, CDEe, $\mathrm{CcDE}, \mathrm{cDE}$, and cDe) prevalence was $14,47 \%$ (Figure 3 ). 


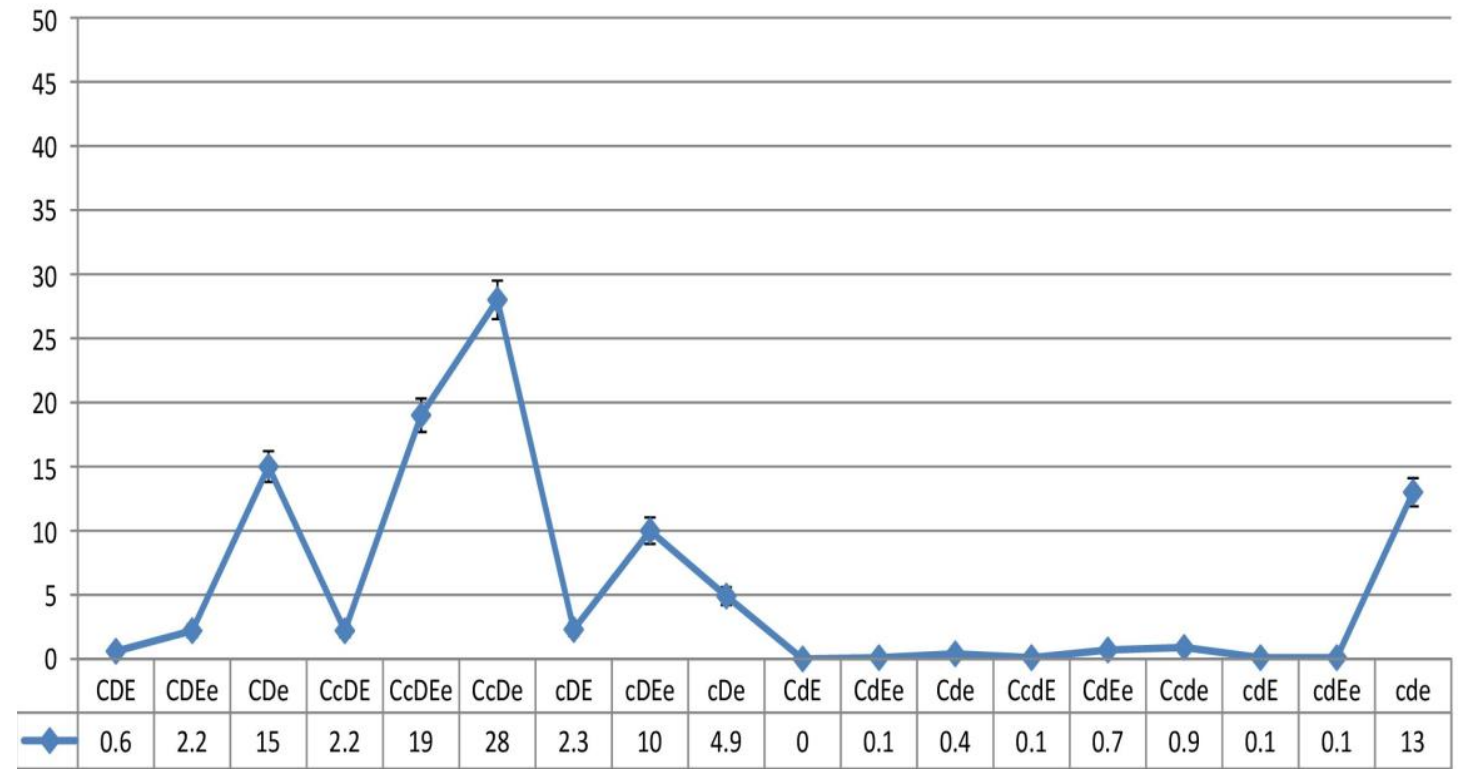

Figure №2. Frequency of Rh blood group phenotypes in studied donors ( $\mathrm{n}=852)$.

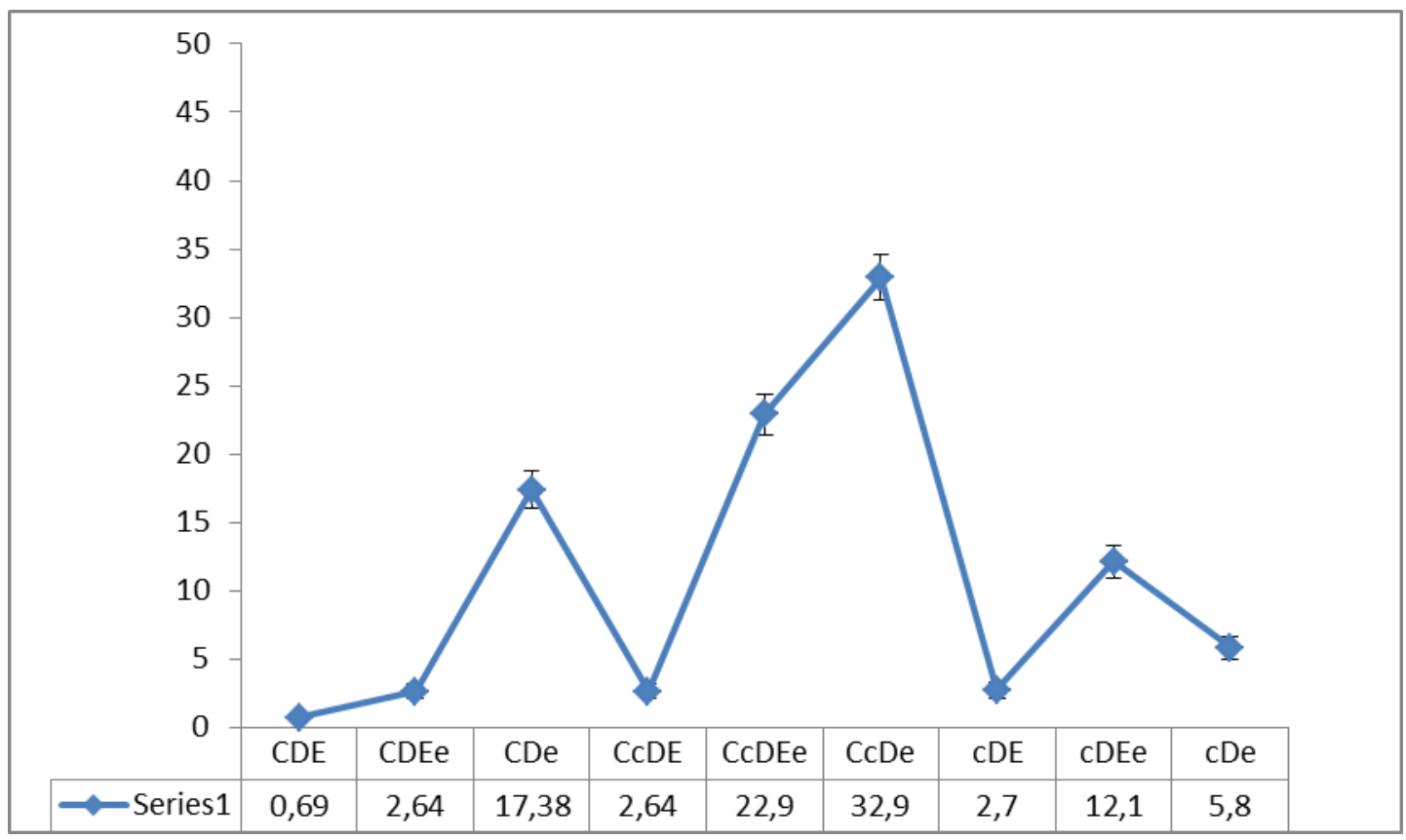

Figure 3. Phenotype variation in Rh positive donors.

Statistically revealed a high number of chi-square criteria, which indicates the unequal distribution of phenotypes. In this particular case, the value $\chi^{2}$ is quite effective for rejecting the null hypothesis $(\mathrm{E}=0)$. The value of $\chi 2$, in this case, is equal to
651. This is much higher than the critical value $(\mathrm{CV})$ of the criterion of the degree of freedom (d.f. $=8$ ), which is equal to 15,51 . The P-Value is $<.00001$. The result is significant at $\mathrm{p}<$ .05 (Table IV). 
Table IV. Rh positive phenotypes chi-square analysis of proportions

\begin{tabular}{|c|c|c|c|c|c|}
\hline $\begin{array}{l}\text { Rh positive } \\
\text { phenotype }\end{array}$ & $(\mathrm{O}-\mathrm{E})^{2} / \mathrm{E}$ & $\mathrm{Df}$ & $\chi^{2}$ & $\mathrm{CV}$ & $\mathbf{P}$ \\
\hline $\mathrm{CDE}$ & 70,11 & \multirow[t]{9}{*}{8} & \multirow[t]{9}{*}{651} & \multirow[t]{9}{*}{15,51} & \multirow{9}{*}{$\begin{array}{c}\text { The P-Value } \\
\text { is }<.00001 \text {. } \\
\text { The result is } \\
\text { significant at } \\
\mathrm{p}<.05 \text {. }\end{array}$} \\
\hline CDEe & 46,32 & & & & \\
\hline $\mathrm{CDe}$ & 25,6 & & & & \\
\hline $\mathrm{CcDE}$ & 46,32 & & & & \\
\hline CcDEe & 90,96 & & & & \\
\hline CcDee & 309 & & & & \\
\hline $\mathrm{cDE}$ & 44,21 & & & & \\
\hline cDEe & 0,65 & & & & \\
\hline ccDee & 17,9 & & & & \\
\hline
\end{tabular}

All donors which blood doesn't show agglutination reaction with anti-D antibodies belong to $\mathrm{Rh}$ negative group. $\mathrm{Rh}$ negative group is less polymorphic in our donors. In contrast to Rh-positive donors in the case of Rh-negative dominant phenotypically characteristics were only one. This is cde phenotype. Totally in the studied donors, we had $143 \mathrm{Rh}-$ negative donors, among them 112 donors had cde phenotypes. We can say that this phenotype is more common for Rh- negative blood donors. The prevalence of this phenotype was $84,2 \%$. Three phenotypes (Ccde, CcdEe, and Cde) prevalence was 7,8 times less than cde phenotype and totally was $12,77 \%$ (Ccde- $6,01 \%$; CcdEe - 4,51\% and Cde $-2,25 \%)$. What about the other four phenotypes (CddEe $0,75 \%$, CcdE- 0,75 , cdE - 0,75\%, cdEe- $0,75 \%$ ) total frequency in the studied donors was only $3 \%$ (Figure 4 ).

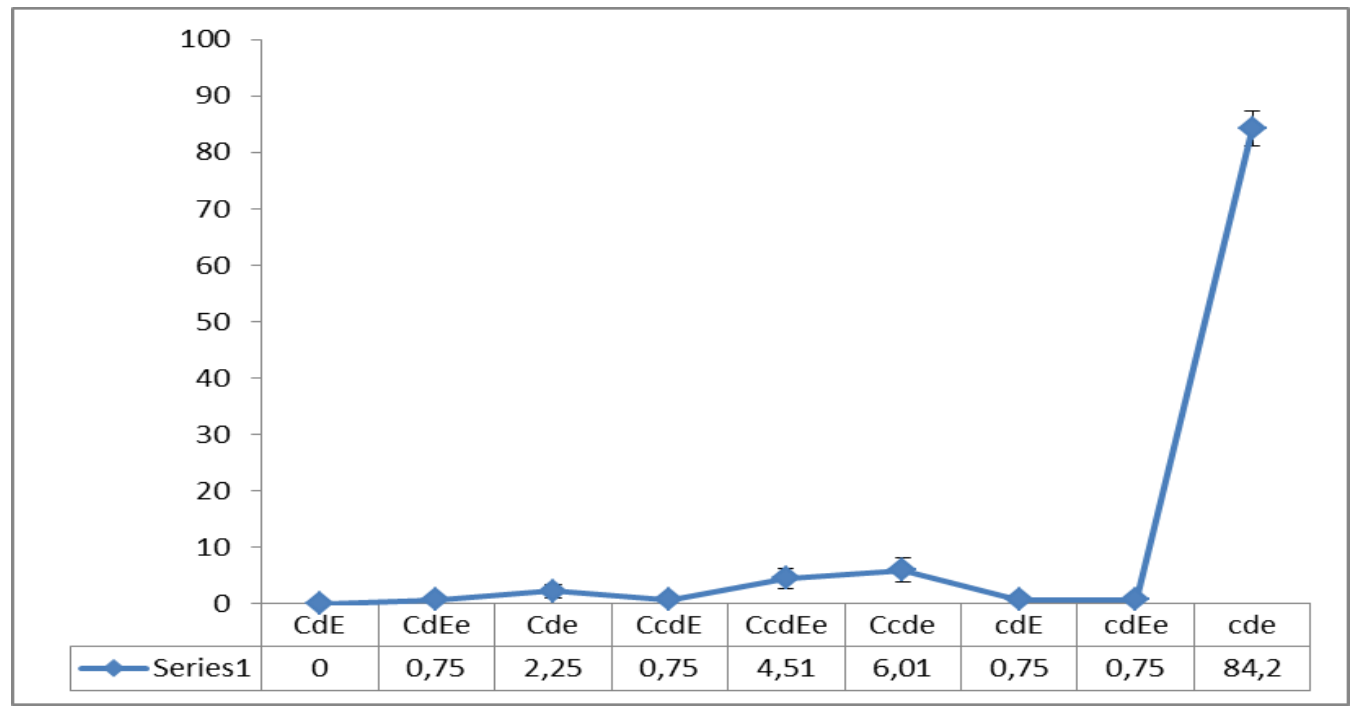

Figure № 4. Phenotype variation in Rh negative donors.

Table № V. Rh negative phenotypes chi-square analysis of proportions.

\begin{tabular}{|c|c|c|c|c|c|}
\hline $\begin{array}{l}\text { Rh negative } \\
\text { phenotype }\end{array}$ & $(\mathbf{O}-\mathrm{E})^{2} / \mathrm{E}$ & Df & $\overline{\chi^{2}}$ & $\overline{C V}$ & $\mathbf{P}$ \\
\hline $\mathrm{CdE}$ & 14,7 & \multirow[t]{9}{*}{8} & \multirow{9}{*}{727} & \multirow{9}{*}{15,51} & \multirow{9}{*}{$\begin{array}{l}\text { The P-Value } \\
\text { is }<.00001 \text {. } \\
\text { The result is } \\
\text { significant at } \mathrm{p} \\
<.05 \text {. }\end{array}$} \\
\hline $\mathrm{CdEe}$ & 12,76 & & & & \\
\hline Cde & 9,31 & & & & \\
\hline $\mathrm{CcdE}$ & 12,76 & & & & \\
\hline CcdEe & 5,1 & & & & \\
\hline Ccde & 3,05 & & & & \\
\hline $\mathrm{cdE}$ & 12,76 & & & & \\
\hline cdEe & 12,76 & & & & \\
\hline Cde & 644 & & & & \\
\hline
\end{tabular}


This case statistically revealed a high number of chi-square criteria and it is equal to 727 . This is much higher than the critical value $(\mathrm{CV})$ of the criterion of the degree of freedom (d.f.=8), which is equal to 15,51 . The P-Value is $<.00001$. The result is significant at $\mathrm{p}<.05$ (Table 5).

The haplotypes of the Rh system were calculated by us in the studied donors. We used the specially formulas for calculation of $\mathrm{Rh}$ haplotypes. It was described above. Theoretically, there are seven haplotypes. All of them were isolated in the target group. They are: cde, Cde, cdE, cDe, cDE, CDe, CDE. Among them, the cde haplotype is most often present in donors and is equal to 0.33 . The lowest frequency of distribution shows the CDE haplotype (Table 6).

Table № VI. Haplotypes of the Rhesus system in the studied donors

\begin{tabular}{|c|c|c|}
\hline Rh haplotypes & Calculated formula & Frequency of haplotypes \\
\hline$C d e$ & $c d e=\sqrt{c c d d e e}$ & 0,33 \\
\hline$C d e$ & $C d e=\frac{C c d d e e}{2 c d e}$ & 0.1 \\
\hline$c d E$ & $c d E=\frac{c c d d E e}{2 c d e}$ & 0.1 \\
\hline$c D e$ & $c D e=\frac{c c D e e}{2 c d e}$ & 0.13 \\
\hline$c D E$ & $c D E=\sqrt{c c D E E+c d E^{2}}$ & 0,23 \\
\hline$C D e$ & $C D e=\sqrt{C C D e e+C d e^{2}}$ & 0.1 \\
\hline$C D E$ & $\frac{C C D E e}{2(C D e+c d e)}$ & 0.02 \\
\hline & $C D E=\frac{}{2}$ & \\
\hline
\end{tabular}

\section{DISCUSSION}

In our work we analyzed the combination of $\mathrm{ABO}$ blood groups and Rh phenotypes. We allocated eight phenotypically groups with a combination $\mathrm{ABO}$ blood group and $\mathrm{D}$ positive and $\mathrm{D}$ negative groups. The above mentioned phenotypically groups were: O (I), Rh+; O (I), Rh-; A (II), Rh+; A (II), Rh-; B
(III), Rh+; R (III), Rh-; AB (IV), Rh +; AB (IV), Rh-. As it is shown from figure № 5 majority $(41,19 \%)$ of the studied donors were $\mathrm{O}(\mathrm{I}), \mathrm{Rh}+(\mathrm{n}=351) .32,7 \%$ donors belonged to the $\mathrm{A}$ (II), $\mathrm{Rh}+$ phenotypically group. The frequency of $\mathrm{B}$ (III), $\mathrm{Rh}+$ phenotypes was $7,74 \%$. The less frequently $(2,69 \%)$ from Rh-positive phenotypes is $\mathrm{AB}(\mathrm{IV}), \mathrm{Rh}+$ (Figure 5). 


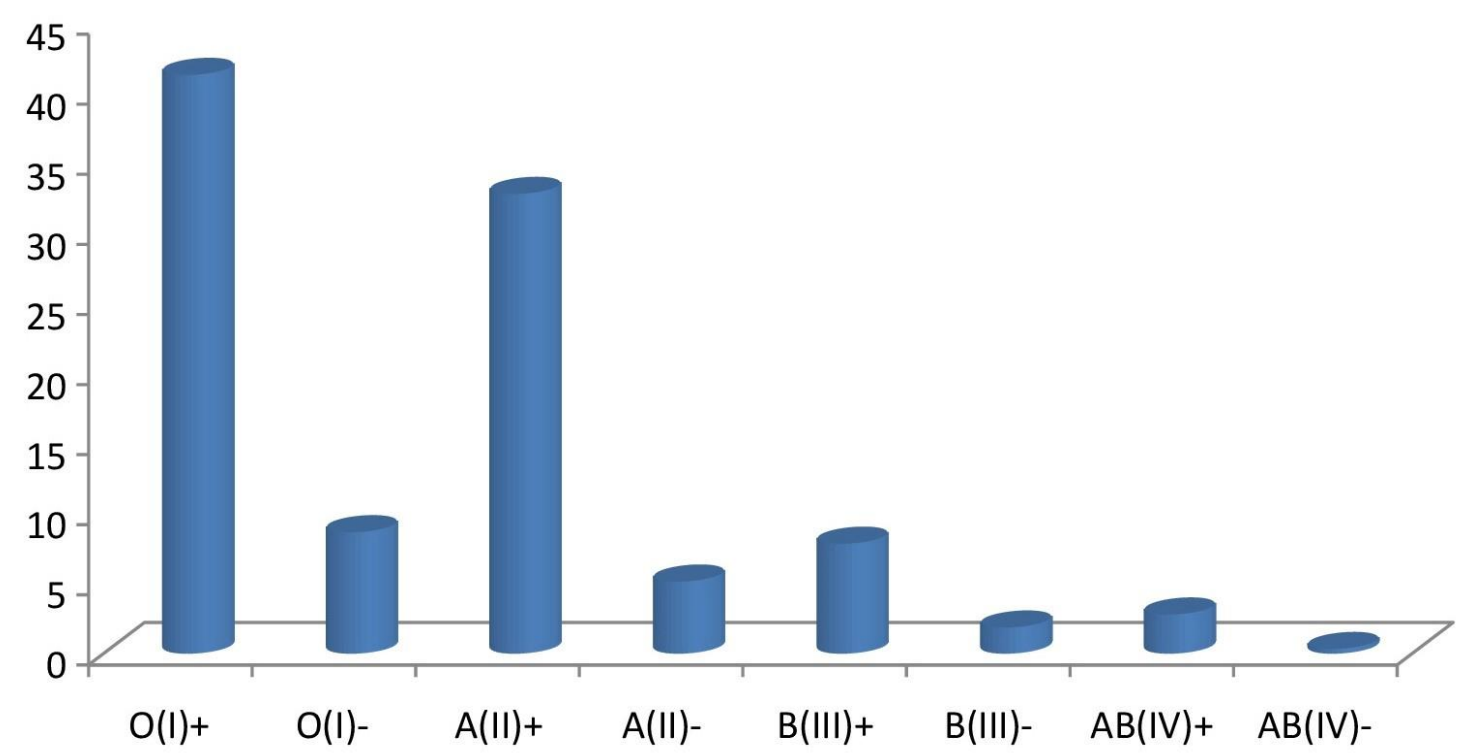

Figure № 5. Frequency of ABO and Rh blood group phenotypes in studied donors $(\mathrm{n}=852)$.

Today in our clinics two antigens (A, B) of ABO system and D antigens for the $\mathrm{Rh}$ system are taken into account during a blood transfusion. For the individuals where those antigens do not occur the theoretical risk of alloimmunosensibilization is high. In the viewpoint of transfusion $\mathrm{c}$ antigen is also significant. Numerous data about alloimmunosensibilization caused by this antigen are presented in the scientific literature [8] [22] [23].

The distribution frequency of $\mathrm{c}$ antigens within the world population is $80-82 \%$. $18-20 \%$ of humans doesn't have this antigen and are revealed in $\mathrm{CC}$ state. Individuals with just this genotype belong to a high-risk group of alloimmunosensibilization caused by c antigen.

We took our attention to cc and Cc genotypic donors because in both cases their erythrocyte membrane contains rhesus system c antigens. As we see in our study frequency of cc genotype in the studied donors is $30,72 \%(\mathrm{ccD}+17,42 \%$ and ccD- 13,3\%), Cc genotype frequency was more high and equals 50,82 (ccD+ - 49,06\% and CcD- - 1,76\%). As we discussed above the recipient who has $\mathrm{CC}$ genotype the immunosensibilization by anti-c antibodies is higher. The frequency of CC genotype in studied donors was $17,88 \%$ (Table №VII).

Table №VII . CC, Cc,cc, EE, Ee, ee phenotypes combination with Rh+ and Rh- case.

\begin{tabular}{|c|c|c|}
\hline & $\mathrm{Rh}+$ & $\mathrm{Rh}-$ \\
\hline $\mathrm{CC}$ & $17,42 \% \pm 1,29$ & $0,46 \% \pm 0,2$ \\
\hline $\mathrm{Cc}$ & $49,06 \% \pm 1,7$ & $1,76 \% \pm 0,4$ \\
\hline $\mathrm{Cc}$ & $17,42 \% \pm 1,29$ & $13,3 \% \pm 1,1$ \\
\hline $\mathrm{EE}$ & $5,1 \% \pm 0,7$ & $0,23 \% \pm 0,1$ \\
\hline $\mathrm{Ee}$ & $31,8 \% \pm 1,5$ & $0,9 \% \pm 0,3$ \\
\hline $\mathrm{Ee}$ & $47,44 \% \pm 1,7$ & $14,43 \% \pm 1,2$ \\
\hline
\end{tabular}

In our previous work we studied the distribution of these genotypes in the Adjara population [24]. They are potential recipients. The distribution frequency of $\mathrm{CC}$ genotype in the Adjara population was equaled to $8 \%$. Implying that carriers of this genotype don't consist in c antigen and during transfusion only $17,88 \%$ of the cases, they received the blood from $\mathrm{CC}$ donors. In the majority of cases, they are at high risk of immunosensibilization by anti-c antibodies because theoretically $82,12 \%$ of cases, they received the blood from
$\mathrm{Cc}$ and $\mathrm{cc}$ donors. The immunization risk by anti-c antibodies is $82,2 \%$ cases. Only $17,88 \%$ of the cases of transfusion with CC genotypic donors are safe.

We have found differences in the distribution of $\mathrm{Rh}$ phenotypes between blood donors and Adjara population, for instance, there are a more phenotypic variation among blood donors than the Adjara population (19). Rh haplotype in the current study is 7 , while in the Adjara region population we fixed only 3 haplotypes (Table VIII). 
Table VIII. Rh haplotype in blood donors and Adjara population.

\begin{tabular}{|c|c|c|}
\hline Rh haplotype & Blood donors & Adjara population \\
\hline$c d e$ & 0,33 & 0,42 \\
\hline$C d e$ & 0.1 & 0 \\
\hline$c d E$ & 0.1 & 0 \\
\hline$c D e$ & 0.13 & 0,11 \\
\hline$c D E$ & 0,23 & 0 \\
\hline$C D e$ & 0.1 & 0,14 \\
\hline$C D E$ & 0.02 & 0 \\
\hline
\end{tabular}

In the same region example of one clinic blood donor, we allocated 2,8 times more Rh phenotypic characteristics (Figure 2 ). We think that this differences reason is that on the study of $\mathrm{Rh}$ antigens in Adjara population level we took our attention on the nationality. All participants were Georgians. In case of blood donors as they are officially donor's nationality is different and donors belong to different ethnic groups.

\section{CONCLUSION}

According to the $\mathrm{Rh}$ blood group antigens and phenotypes our studied donors are characterized by rather high polymorphism. The Georgian donor's population is heterogenic, especially high heterogeneity are shown in Rh-positive phenotypes. Each geographic area has its specific characteristics. Alloimmunization rates caused by Rh blood group antigens are not similar in different geographic area and dependent on the $\mathrm{Rh}$ phenotype of that population. The study was helpful for us to establish a database of clinic donors according to Rh group antigens. In our work, we used serological methods, while a molecular study is much informative. In the future, we are planning to include molecular investigations and also study the donors immunosensibilization with anti-Rh antibodies.

\section{REFERENCE}

[1] M. E. Reid and V. Yahalom, "Blood groups and their function," Best Pract. Res. Clin. Haematol., vol. 13, no. 4 , pp. 485-509, Dec. 2000, doi: 10.1053/beha.2000.0096.

[2] G. Daniels and I. Bromilow, Eds., Essential Guide to Blood Groups: Daniels/Essential Guide to Blood Groups. Oxford: John Wiley \& Sons, 2013.

[3] L. Svensson, Chemical basis of ABO subgroups insights into blood group A subtypes revealed by glycolipid analysis. Göteborg: Department of Clinical Chemistry and Transfusion Medicine, Institute of Biomedicine, The Sahlgrenska Academy at University of Gothenburg, 2011.
[4] O. Wu, N. Bayoumi, M. A. Vickers, and P. Clark, "ABO $(\mathrm{H})$ blood groups and vascular disease: a systematic review and meta-analysis: $\mathrm{ABO}$ groups and thrombosis," J. Thromb. Haemost., vol. 6, no. 1, pp. 6269, Oct. 2007, doi: 10.1111/j.1538-7836.2007.02818.x.

[5] M. Zietz, "Associations between blood type and COVID-19 infection, intubation, and death," p. 6.

[6] I. Nakashidze, Nanuli Kotrikadze, Anzor Diasamidze, Marina Nagervadze, Manana Alibegashvili, Liana Ramishvili and Manana Gordeziani. ERYTHROCYTE BLOOD GROUP ANTIGENS AND ALTERATIONS OF THE HORMONAL STATUS AMONG THE REPRODUCTIVE AGE WOMEN WITH BREAST TUMORS," Eur. Med. Health Pharm. J., vol. 7, no. 1, May 2014, doi: 10.12955/emhpj.v7i1.429.

[7] "[Blood groups - minuses and pluses. Do the blood group antigens protect us from infectious diseases?] PubMed." https://pubmed.ncbi.nlm.nih.gov/26206987/ (accessed Apr. 01, 2021).

[8] C. S. Sheeladevi, S. Suchitha, G. V. Manjunath, and S. Murthy, "Hemolytic Disease of the Newborn Due to Anti-c Isoimmunization: A Case Report," Indian J. Hematol. Blood Transfus., vol. 29, no. 3, pp. 155-157, Sep. 2013, doi: 10.1007/s12288-012-0159-6.

[9] S. Abourazzak, S. Hajjaj, C. Hakima, A. Bouharrou, and M. Hida, "Haemolytic disease of the newborn due to anti-c," Case Rep., vol. 2009, no. feb24 1, pp. bcr0920080987-bcr0920080987, Feb. 2009, doi: 10.1136/bcr.09.2008.0987.

[10] D. Basu, S. S. Datta, C. Montemayor, P. Bhattacharya, K. Mukherjee, and W. A. Flegel, "ABO, Rhesus, and Kell Antigens, Alleles, and Haplotypes in West Bengal, India," Transfus. Med. Hemotherapy, vol. 45, no. 1, pp. 62-66, 2018, doi: 10.1159/000475507.

[11] A. V. Romphruk et al., "Phenotype frequencies of Rh (C, c, E, e), M, Mi a and Kidd blood group systems among ethnic Thai blood donors from the north- east of Thailand," Int. J. Immunogenet., vol. 46, no. 3, pp. 160165, Jun. 2019, doi: 10.1111/iji.12420.

[12] C. T. Hamed and M. A. Bollahi, "Distribution of Rhesus and Kell blood group frequencies in the Mauritanian population," Blood Transfus., 2013, doi: 10.2450/2012.0022-12. 
[13] L. Lasić, N. Lojo-Kadrić, E. Silajdžić, L. Pojskić, R. Hadžiselimović, and N. Pojskić, "Molecular - genetic variance of $\mathrm{RH}$ blood group system within human population of Bosnia and Herzegovina," Bosn. J. Basic Med. Sci., vol. 13, no. 1, p. 10, Feb. 2013, doi: 10.17305/bjbms.2013.2403.

[14] N. D. Avent and M. E. Reid, "The Rh blood group system: a review," Blood, vol. 95, no. 2, pp. 375-387, Jan. 2000, doi: 10.1182/blood.V95.2.375.

[15] R. E. Rosenfield, F. H. Allen, and P. Rubinstein, "Genetic Model for the Rh Blood-Group System," Proc. Natl. Acad. Sci., vol. 70, no. 5, pp. 1303-1307, May 1973, doi: 10.1073/pnas.70.5.1303.

[16] O. Jahanpour, J. J. Pyuza, E. O. Ntiyakunze, A. Mremi, and E. R. Shao, "ABO and Rhesus blood group distribution and frequency among blood donors at Kilimanjaro Christian Medical Center, Moshi, Tanzania," BMC Res. Notes, vol. 10, no. 1, p. 738, Dec. 2017, doi: 10.1186/s13104-017-3037-3.

[17] C. T. Hamed et al., "Frequencies and ethnic distribution of $\mathrm{ABO}$ and $\mathrm{Rh}(\mathrm{D})$ blood groups in Mauritania: results of first nationwide study: Frequencies and ethnic distribution of $\mathrm{ABO}$ and $\mathrm{Rh}(\mathrm{D})$ in Mauritania," Int. J. Immunogenet., vol. 39, no. 2, pp. 151-154, Apr. 2012, doi: 10.1111/j.1744-313X.2011.01064.X.

[18] A. Owaidah, N. Naffaa, A. Alumran, and F. Alzahrani, "Phenotype Frequencies of Major Blood Group Systems (Rh, Kell, Kidd, Duffy, MNS, P, Lewis, and Lutheran) Among Blood Donors in the Eastern Region of Saudi Arabia," J. Blood Med., vol. Volume 11, pp. 59-65, Feb. 2020, doi: 10.2147/JBM.S236834.

[19] "The Blood Group Antigen FactsBook - 2nd Edition." https://www.elsevier.com/books/the-blood-groupantigen-factsbook/reid/978-0-12-586585-2 (accessed Apr. 01, 2021).

[20] "The Rh blood group - Blood Groups and Red Cell Antigens - NCBI Bookshelf." https://www.ncbi.nlm.nih.gov/books/NBK2269/ (accessed Apr. 07, 2021).

[21] W. A. Flegel, "The genetics of the Rhesus blood group system," Blood Transfus., no. 5, pp. 50-57, 2007, doi: 10.2450/2007.0011-07.

[22] A. A. George and C. D. Simon, "Anti-c (Little c) IgM: An Uncommonly Observed but Expected Phenomenon," Lab. Med., vol. 45, no. 4, pp. e142-e145, Nov. 2014, doi: 10.1309/LME8U8K5GVGWBAOY.

[23] D. N. Hackney, E. J. Knudtson, K. Q. Rossi, D. Krugh, and R. W. O'Shaughnessy, "Management of Pregnancies Complicated by Anti-c Isoimmunization:," Obstet. Gynecol., vol. 103, no. 1, pp. 24-30, Jan. 2004, doi: 10.1097/01.AOG.0000109206.22354.2C.

[24] M. Nagervadze, A. Diasamidze, L. Akhvlediani, G. Dumbadze, R. Khukhunaishvili, M. Koridze and S. Tckvitinidze. "Distribution of $\mathrm{ABO}$ and $\mathrm{Rh}-\mathrm{Hr}$ blood group antigens, alleles and haplotypes in the mountain region of Ajara (Georgia) . African Journal of Biotechnology. Vol. 10, no 38, pp. 7324-7329, July, 2011. DOI: $10.5897 / A J B 10.1395$

\section{Creative Commons Attribution License 4.0 (Attribution 4.0 International, CC BY 4.0)}

This article is published under the terms of the Creative Commons Attribution License 4.0 https://creativecommons.org/licenses/by/4.0/deed.en_US 